\title{
EL CONCEPTO DE LEBENSWELT EN HUSSERL ${ }^{1}$
}

\author{
por Dagfin Follesdal \\ (Traducción de Sergio Sánchez Benítez)
}

La idea husserliana que ha adquirido una mayor popularidad es, sin duda, la del mundo de la vida. En concreto, la expresión 'mundo de la vida', o 'Lebenswelt', ha alcanzado una amplia difusión. Esta expresión fue usada por Simmel y otros antes de Husserl ${ }^{2}$. Después de la II Guerra Mundial se convirtió en la palabra favorita de muchos cientificos sociales que la usaron con multitud de significados diferentes. Muchos aludían a Husserl sin que parezca que hubieran estudiado su filosofía y, por ello, sin ser conscientes de los muchos importantes matices que el mundo de la vida tiene en la obra de Husserl.

La primera obra publicada por Husserl que contiene la palabra Lebenswelt es su Krisis, cuyas dos primeras partes fueron publicadas en 1936. El resto de la obra inacabada, incluyendo la importante tercera parte con la discusión capital acerca del mundo de la vida, no fue publicada hasta 1954; pero fue conocida por algunos estudiosos y seguidores de Husserl, entre ellos Maurice Merleau-Ponty quien llegó en abril de 1939 al Archivo Husserl en Lovaina con el fin de estudiar dicha parte ${ }^{3}$.

Los intérpretes de Husserl difieren entre ellos enormemente en sus opiniones respecto al mundo de la vida. Se piensa a menudo que este concepto constituye la mayor interrupción en el desarrollo de la filosofía husserliana desde el "primer" Husserl de Ideas al último de La crisis. ¿Podemos hablar de tal interrupcion? Y, en segundo lugar, ¿qué es exactamente el mundo de la vida y qué papel juega en la 
fenomenologfa? Mi contestación a la primera de las cuestiones es un contundente "No". En lo sucesivo argumentaré que el mundo de la vida es totalmente compatible con la primera filofia husserliana y que incluso hay ya desde sus comienzos un lugar preciso para ella en su fenomenologia. En repetidas ocasiones, Husserl hizo breves alusiones sobre el mundo de la vida en sus primeros trabajos y gradualmente fue profundizando y modificando sus puntos de vista sobre este concepto, como acostumbraba a hacer siempre con todo las piezas de su fenomenologia. Sin embargo, éste estuvo siempre presente.

En lugar de considerar el mundo de la vida como una ruptura con la primera filosoffa husserliana, deberfamos observar la problemática del mundo de la vida como íntimamente conectada con otros temas fundamentales de la fenomenologia, en especial, con el de la intencionalidad. Para comprender apropiadamente el mundo de la vida con todos sus matices es importante apreciar la total conexión entre este y el resto de la filosoffa husserliana. Para contestar nuestra segunda pregunta, acerca de qué es el mundo de la vida y cuál es su papel en la fenomenología, empezaré con una exposición de las ideas clave de la fenomenología husserliana, para pasar a demostrar que el mundo de la vida es una parte integrante de su fenomenologla desde los comienzos de ésta.

\section{LA FENOMENOLOGIA COMO ESTUDIO DE LA PERPECTIVA SUBJETIVA}

La fenomenologla pretende estudiar de modo sistemático nuestras diferentes perspectivas subjetivas, nuestras diferentes maneras de experimentar la realidad. En las ciencias se busca objetividad: se trata de asegurar que las observaciones de alguien son independientes 
de aquel que las lleva a cabo. Estas deberfan consistir a ser posible en la lectura de números en una escala de medida o en cualquier otro tipo de registro que se dejara afectar lo menos posible por la perspectiva subjetiva del observador. No se niega con esto la existencia de una perspectiva subjetiva, pero se la considera como un elemento molesto cuando se efectuan observaciones cientfficas. Se trata, por tanto, de disponer los experimentos y observaciones de modo que la influencia de la perspectiva subjetiva se reduzca al máximo posiblet.

Cuando nuestro objetivo es comprender a otras personas, no nos basta con conocer a qué estímulos están expuestos y cómo se mueven. Es mucho más importante conocer cómo se experimentan a ellos mismos y a su entorno. Para comprender lo que Husserl nos dirá despues al respecto, podemos comenzar con un ejemplo sencillo, el dibujo pato/conejo ideado por el psicólogo Jastrow y popularizado por Wittgenstein. En realidad, para acercarnos a la posición de Husserl tenemos que modificar el ejemplo y considerar no un dibujo sino la silueta de un animal real con el cielo como fondo:

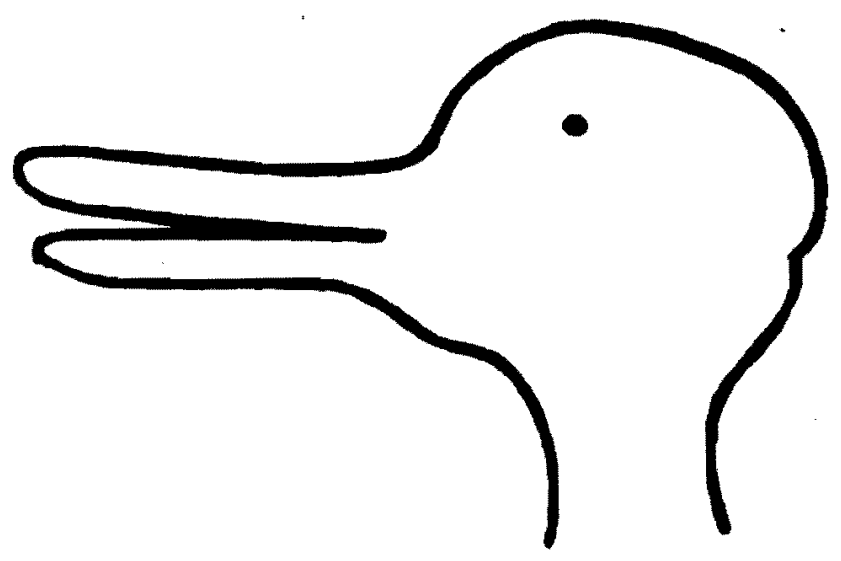


Cuando vemos esta silueta con el cielo como fondo podemos ver un pato o un conejo. Lo que alcanza nuestros ojos es lo mismo en ambos casos, luego la diferencia debe estribar en algo que proviene de nosotros. Estructuramos aquello que vemos, y podemos hacerlo en diferentes maneras. Los impulsos que llegan hasta nosotros desde el exterior son insuficientes por sí solos para determinar cuál es el objeto que estamos experimentando, así que algo más se añade a estos.

\section{EL NOEMA, INTENCIONALIDAD}

Este algo más que se añade es denominado noema por Husserl. El noema es un estructura. Nuestra conciencia estructura aquello que que experimenta. El modo en el qué estructuramos depende de nuestras experiencias previas, del conjunto todo de nuestra experiencia presente y de otros factores más. Si nos hemos criado rodeados de patos, pero jamás hemos ni tan siquiera oido a un conejo, deberíamos estar más predispuestos a ver un pato que un conejo cuando nos enfrentamos con la silueta de arriba; probablemente, incluso, no se nos ocurriría la idea de un conejo. De acuerdo con Husserl, nuestra experiencia en una situación determinada puede ser, en principio, estructurada siempre de diferentes maneras; aquello que alcanza nuestros sentidos no es nunca suficiente para determinar lo que experimentamos. Sólo muy rara vez, como en el ejemplo del pato/conejo, podemos ir hacia adelante y saltar hacia atrás a voluntad entre diferentes modos de estructurar nuestra experiencia. Por regla general, no tenemos incluso conciencia de que se esté llevando a cabo ninguna estructuración, sino que experimentamos objetos como teniendo una estructura. 
La estructuración acaece siempre de modo que las múltiples características diferentes del objeto terminan vinculándole con otro y se experimentan como características de un único y mismo objeto. Cuando, por ejemplo, vemos un conejo, no vemos sólo una colección de manchas coloreadas, varias tonalidades de marrón esparciéndose en nuestro campo visual. Nosotros vemos un conejo con una determinada forma y un determinado color, con capacidad para comer, saltar, etc. Uno de sus lados mira hacia nosotros y el otro en dirección opuesta. No vemos el otro lado desde donde nosotros nos hallamos, pero lo vemos como teniendo otro lado. Husserl expresa esto diciendo que nuestra conciencia se caracteriza por su intencionalidad; está siempre dirigida hacia un objeto. Que ver es intencional o dirigido a un objeto significa precisamente que el lado próximo del objeto que tenemos enfrente se observa como el lado de una cosa que tiene otros lados y características que son también cointencionadas, en el sentido de que la cosa es observada como algo más que sólo este único lado. El noema es el sistema comprehensivo de determinaciones que confiere unidad a esta multitud de características y hace de ellas aspectos de uno y el mismo objeto.

La palabra 'objeto' tiene que tomarse aqui en sentido lato, comprendiendo no sólo cosas físicas sino también, como hemos visto, animales y, del mismo modo, personas, eventos, acciones y procesos. Cuando experimentamos una persona, no experimentamos un objeto físico, un cuerpo, y entonces inferimos que una persona está ahr. Experimentamos una persona hecha y derecha, nos tropezamos con alguien que estructura el mundo y lo experimenta desde su propia perspectiva. Nuestro noema es el noema de una persona sin que medie ninguna inferencia. Ver personas no es más misterioso que ver objetos f́sicos, no se precisa ninguna inferencia en ninguna de estos casos. Cuando vemos un objeto físico no vemos sensa data o algo similar y 
entonces inferimos que se trata de un objeto físico; nuestro noema es el de un objeto físico. De modo similar, cuando vemos una acción lo que vemos es una acción en sentido propio no un movimiento corporal del cual inferimos que hay tal acción.

La noción de noema es clave en la fenomenología de Husserl, no sólo en su teorfa de la percepción sino también en su análisis de todos los aspectos de la conciencia humana. El noema representa, precisamente, el intento husserliano de caracterizar la perpectiva subjetiva. Dos personas pueden hacer frente a la misma cosa pero, sin embargo, tener una experiencia distinta de ellas; tan diferentes como un pato y un conejo. Pero, incluso, cuando estamos de acuerdo respecto al tipo de objeto del que se trata puede haber enormes diferencias entre las formas de verlo. Todos caemos en formas rutinarias de experimentar el mundo circundante y a nosotros mismos. Lo que caracteriza a un gran artista es, en parte, la capacidad para ver y experimentar cosas, eventos y personas en diferentes maneras; en parte, la capacidad para transmitir esta experiencia a otros. Aquellos objetos que se experimentan no necesitan ser nuevos ni excepcionales. Son, a menudo, ordinarios y comunes. No es el objeto, sino la experiencia, lo que es central.

\section{CUMPLIMIENTO}

En el caso de un acto de percepción, su noema puede ser también caracterizado como un conjunto muy complejo de expectaciones o anticipaciones acerca del tipo de experiencias que tendremos cuando nos movamos en torno al objeto y lo percibamos usando nuestros distintos sentidos. Cuando vemos un pato anticipamos diferentes experiencias posteriores que cuando vemos un conejo. En 
el primer caso, anticipamos, por ejemplo, que sentiremos plumas cuando toquemos el objeto; en el segundo caso, esperamos encontrar piel. Cuando obtenemos las experiencias que habfamos anticipado, se dice que el correspondiente componente del noema ha sido cumplido. En toda percepción se dará algún cumplimiento: los componentes del noema que corresponden a lo que en este momento "encuentra el ojo" se cumplen, de modo similar para el resto de los sentidos.

Tal anticipación y cumplimiento es lo que distingue a la percepción de otros modos de conciencia, por ejemplo, imaginación o recuerdo. Si meramente imaginamos cosas, nuestro noema puede serlo de cualquier cosa; un elefante o una locomotora que están aquí a mi lado. En la percepción, sin embargo, se ven involucradas mis experiencias sensoriales. Esto elimina un cierto número de noemata que yo no hubiera tenido en el caso de estar solamente imaginando. En mi situación actual no podría tener un noema correspondiente a la percepción de un elefante.

Esto no reduce a uno solo el número de noemata perceptuales que yo puedo tener, por ejemplo, de ti sentado alli en frente mia. Es un punto central en la fenomenología husserliana que yo pueda tener una variedad de diferentes noemata perceptuales que son compatibles con las impresiones presentes en mis receptores sensoriales. En el caso del pato/conejo esto era evidente, nosotros podramos ir hacia a un lado u otro del dibujo a voluntad teniendo ora el noema de un pato, ora el noema de un conejo. En la mayoría de los casos, sin embargo, no somos conscientes de esta posibilidad. Sólo cuando ocurre algo adverso, cuando me encuentro con una experiencia "recalcitrante" que no encaja con las anticipaciones de mi noema, empiezo a ver un objeto diferente de aquel que había pensado ver. Mi noema "explota", usando la expresión husserliana, y tengo entonces un noema bastante distinto de aquel que previamente habla tenido, y con nuevas 
anticipaciones. Esto es siempre posible, dice Husserl. La percepción supone siempre anticipaciones que van más allá de lo que actualmente "encuentra el ojo", y hay siempre riesgo de fracaso independientemente de lo fiel y certeramente que podamos experimentar, La percepción errónea es siempre posible.

\section{CONSTITUCION}

Apuntamos intencionalmente a los objetos como teniendo un gran número de propiedades, normalmente, como en el caso de un objeto material, muchas más de las que pueden agotarse en nuestra experiencia de él. Según Husserl, los objetos se constituyen mediante nuestra conciencia. Esto no significa que nosotros los creemos o que los produzcamos, sino solo que los diversos componentes del noema están interconectados de tal modo que tenemos la experiencia de que se trata de un objeto completo. Todo lo que hay en el objeto corresponde, por tanto, a los componentes de nuestro noema. En el caso de objetos físicos, el carácter inagotable de lo que es experimentado es una anticipación característica en nuestro noema y este es un rasgo importante de lo que es objeto físico.

De acuerdo con Husserl,

se "constituye" un objeto -"sea real o no"- en ciertos complejos de conciencia que ostentan una unidad evidente en la medida que llevan esencialmente en sí la conciencia de una $\mathrm{X}$ idéntica. ${ }^{5 *}$

* N. del T. Aqui y en lo sucesivo se cita la traducción de José Gaos en E. HUSSERL, Ideas, F.C.E., 1* reimpresión en España, Madrid, 1985. El resto de las traducciones son mías. 
La utilización por Husserl, aquf y en muchos otros lugares, de la forma reflexiva 'se constituye un objeto' es, incidentalmente, una indicación de que él no observaba el objeto como siendo producido por la conciencia. Husserl consideraba la fenomenologfa como la primera versión estrictamente cientifica del idealismo transcendental, pero también mantuvo que la fenomenología transciende la distinción tradicional realismo-idealismo. En 1934 escribió en carta a Abbe Baudin: "Ningún 'realista' ordinario ha sido todavia tan realista y concreto como yo, el 'idealista' fenomenológico (una palabra que, por cierto, ya no uso más)". ${ }^{6}$

En el prefacio a la edición inglesa de Ideas, Husserl afirmaba:

El idealismo fenomenológico no niega la existencia de hecho [wirklich] del mundo real [real] como si se tratase de un ilusión... Su única tarea y acierto estriba en clarificar el sentido [Sinn] de este mundo, exactamente ese sentido en el cual todos nosotros todos nosotros lo consideramos como existiendo realmente y como realmente válido. Que el mundo existe...es bastante indudable. Otra cosa es comprender esta certeza que es la base de la vida y la ciencia y aclara la base para su reivindicación. $^{7}$

\section{EL MUNDO, EL PASADO Y LOS VALORES}

No sólo constituimos las diferentes propiedades de las cosas sino también la relación de la cosa con otros objetos. Si, por ejemplo, veo un árbol, este es concebido como algo que está en frente mía, quizás como algo que está situado entre otros árboles, visto por otra gente a parte de mi, etc. Es también concebido como algo que tiene una historia: estuvo ahr antes de que yo lo viera y permanecerá después de que yo me vaya, quizás sea cortado y transportado a algún otro lugar. Sin embargo, como todas las cosas materiales, no desparecerá simplemente del mundo. 
Mi conciencia del árbol se convierte aś también en conciencia del mundo en el espacio y en el tiempo en el cual se situa el árbol. Mi conciencia constituye el árbol; pero, al mismo tiempo, constituye el mundo en el cual, tanto el árbol como yo, vivimos. Si mi próxima experiencia me obliga a a abandonar la creencia de que yo tengo un árbol frente a mí porque, por ejemplo, no encuentro nada parecido a un árbol alrededor o porque alguna otra de mis expectativas no se ha cumplido, esto afectaría no sólo a mi concepción de lo que hay sino también de lo que ha sido y será. Por ello en este caso, yo no reconstituyo sólo el presente sino también el pasado y el futuro. Para ilustrar como cambia mi percepción presente, conduciéndome a la reconstitución no sólo del pasado sino también del futuro, Husserl usa el ejemplo de un balón que yo tomé por rojo y completamente esférico. Cuando lo giro, descubro que era verde por el otro lado y que tiene un agujero:

...el sentido de la percepción no sólo se modifica en la nueva extensión momentánea; la modificación noemática fluye en la forma de una cancelación retroactiva en la esfera retencional y modifica la producción del sentido fluyendo desde fases más primitivas de la percepción. La apercepción más primitiva, que estaba en consonancia con el desarrollo armonioso de lo "rojo uniformemente redondo" es implícitamente "reinterpretado" a "verde por uno de los lados y mellado"

Hasta ahora he mencionado sólo las propiedades de las cosas. Pero, según Husserl, sus propiedades valorativas se constituyen de manera análoga. El mundo en el que vivimos es experimentado como un mundo en el cual ciertas cosas y acciones tienen un valor positivo, otras negativo. Nuestras normas y valores son también susceptibles de cambio. Cambios en nuestros puntos de vista acerca de cuestiones de hecho son, a menudo, acompañadas por cambios en nuestras evaluaciones. 


\section{HORIZONTE}

Mientras experimentamos un objeto, nuestra conciencia está focalizada sobre este objeto; el resto del mundo con su diversos objetos está almacenado en nuestro repertorio como algo "en lo que creemos" pero a lo que no prestamos atención en este momento. Lo mismo es válido para la mayoría de las múltiples características inagotables del objeto mismo. Todás estas características adicionales del objeto, junto con el mundo que lo envuelve, constituye lo que Husserl llama el horizonte de esa experiencia. A las diversas características del objeto, que son cointencionadas, o también mentadas, pero no en el foco de nuestra atención, las llama Husserl el horizonte interno mientras que el reino de los otros objetos y el mundo al que pertenecen lo denomina horizonte externo:

Por eso, cada experiencia de una cosa particular tiene su horizonte interno, y entiendo aquí por "horizonte" la inducción que pertenece esencialmente a cada experiencia y es inseparable de ella siendo to mismo en la experiencia. El término "inducción" es útil porque sugiere [vordeutet] (él mismo una "inducción) induccion en el sentido ordinario de un modo de inferencia y también porque implica que este último, para que su elucidación sea completamente inteligible, tiene que referirse nuevamente a la anticipación originaria básica.

Sin embargo, este dirigirse-más-allá (Hinausmeinen) no es sólo la anticipación de determinaciones que, en la medida en que pertenecen a este objeto de experiencia, se esperan ahora; en otro sentido, es también un dirigirse-más-allá de la cosa misma... hacia otros objetos de los cuales tenemos conciencia al mismo tiempo, aunque en un primer momento estaban meramente en el repertorio. Esto significa que cada cosa dada en la experiencia tiene no sólo un horizonte interno sino también un horizonte externo infinito, abierto, de objetos co-dados... Estos son objetos a los que ahora no me he dirigido, pero a los que me puedo volver en cualquier momento... Todas loas cosas reales que están anticipadas en cualquier momento dado de un modo conjunto o 
co-dadas sólo en el trasfondom como un horizonte externo son conocidas como objetos rreales (o propiedades, o relaciones etc.) del mundo, son conocidas como existiendo dentro del horizonte espaciotemporal. ${ }^{9}$

Tomemos un ejemplo simple de un f́tem que pertenece a este horizonte externo. Si yo te hubiera preguntado cuál eran tus expectativas cuando entrabas en esta habitación, tú podías haber mencionado algo sobre amigos que esperabas encontrar, una conferencia que esperabas oir, y así sucesivamente. Pero incluso, como te he visto entrar caminando confiadamente, tengo muy buenas razones para pensar que además esperabas que hubiera suelo en la habitación. Tú no habías pensado en esto, tu intención se dirigía hacia otras cosas pero tú tenías una disposición para actuar así. $Y$, entonces, si te hubiera preguntado si esperabas que hubiera suelo en la habitación, podrías haberte sorprendido del porqué de una pregunta tan trivial pero seguramente hubieras contestado que sí.

Expectativas y creeencias son nociones disposicionales. Incluimos bajo las creeencias no sólo a pensamientos que nos ocupan activamente, sino también aquellos sobre los que raramente nos paramos a pensar, por ejemplo; que $2+2=4$. No encontramos con un problema cuando tratamos de delimitar exactamente nuestras creeencias. Preguntar por ellas no es un método fiable. Por un lado, concede demasiado, recordemos como en el Menon un interrogador adiestrado descubre que el joven esclavo tenía los más insospechadas creencias sobre geometría. Platón lo tomaba como una evidencia para su teoría de la anámnesis. Por otro lado, cede demasiado poco; como Freud y otros nos han enseñado, a menudo negamos sinceramente que tenemos creencias que, por lo visto, parecen controlar totalmente nuestras acciones. 
El criterio más fiable al que a menudo recurrimos, consiste en asumir que la gente posee aquellas creencias que mejor explican sus acciones, incluyendo sus actividades verbales. Sin embargo, el siguiente problema estriba en que los los estados a los que recurrimos para explicar las acciones de la gente no son exclusivamente cognitivos. Es necesario recurrir también a estados físicos y habilidades de diversos tipos que serfa dif́cil clasificar como mentales. Puesto que, mientras nuestras prácticas aritméticas son, presumiblemente, mentales, nadar o andar no se dejan clasificar fácilmente como tales. También contamos con espinosos casos intermedios tales como mantener una distancia convencional entre contertulios durante una conversación, donde la convención puede variar de cultura a cultura. ¿Es mantener esta distancia una cuestión de la creencia tácita de que esta es la distancia apropiada? ¿O se trata de una habilidad corporal que se adquiere gradualmente por el hecho de haber sido educado en esta cultura? ¿Y qué ocurre con nuestro modo de firmar? ${ }^{10}$ Evidentemente, actividades cognitivas concurren en el proceso que nos conduce a firmar como lo hacemos; tenemos que aprender el alfabeto, nuestro nombre, $y$ asi sucesivamente. Pero también, en nuestra semiautomática forma de firmar, se hallan implicadas en gran medida habilidades corporales. Diversos rasgos de la personalidad, as! como ciertos rasgos de nuestra cultura, desempeñan aqui un papel.

Las opiniones acerca de ejemplos como los anteriores pueden diferir, puesto que al carecer de una definición precisa de qué debemos considerar mental, qué f́́sico, carecemos también de un método bien definido para clasificar tales rasgos. Sin embargo, se establece aquf una interacción entre el proceso que conduce a la práctica y la práctica misma; toda teorfa satisfactoria de la intencionalidad debe tener en cuenta tal interaccion. El noema puede ser considerado todavía como una estructura, pero las anticipaciones que 
mantienen una relación con esta estructura no son sólo aquellas de la visión, de la audición y otras similares sino también las de la cinestesia y movimiento corporal en las que somos conscientes de si algo "va mal". Estamos familiarizados con esta experiencia de "ir mal" mediante los casos de percepción errónea: no podemos decir siempre exactamente qué fue lo que fallo, pero somo conscientes de que algo falló.

Como traté de demostrar en mi contribución al Festschrift dedicado a Hintikka por su $50^{\circ}$ aniversario ${ }^{11}$, Husserl comenz 6 en Ideas y otras de sus primeras obras con una fuerte actitud cognitivista: las anticipaciones en el noema son de tipo puramente cognitivo. Sin embargo, a partir de manuscritos de 1917 , se empezó a concretar más y más en el papel de lo práctico y del cuerpo en nuestra constitución del mundo. Husserl no llegó a elaborar concienzudamente la importantes implicaciones de esto último para su concepción del noema, pero parece claro que no habrfa considerado nuestras anticipaciones como meras creencias sino también como posiciones corporales que se hallan también involucradas en la cinestesia y también juegan un importante papel en la percepción y en los movimientos de nuestro cuerpo. En numerosos pasajes, algunos de los cuales he citado en este artículo, Husserl habla acerca de anticipaciones prácticas y del papel de la cinestesia en la percepción y la actividad corporal.

\section{EL NOEMA, EL HORIZONTE Y EL MUNDO}

Se ha pensado algunas veces que el noema comprende sólo algunas de las anticipaciones que tenemos en un momento dado, a saber, aquellas que corresponden al objeto que al atendemos y a sus propiedades. El resto de las anticipaciones, que conciernen al resto del 
mundo, no estarian incluidas en el noema. Husserl jamás hizo explícita su posición a este respecto. Sin embargo, no hay base en los escritos de Husserl para una concepción tan restringida del noema. Por el contrario, muchos pasajes apuntan hacia una identificación del noema con el conjunto total de anticipaciones, incluyendo aquellas que tienen que ver con el horizonte externo y el mundo. Por ello, cuando introduce la noción de noema en Ideas, dice:

El mundo transcendente queda "colocado entre paréntesis"; ... Con el mundo entero físico y psíquico, ha quedado desconectada la "verdadera" existencia de la relación real en sentido estricto entre percepción y percepto; $y$, sin embargo, ha quedado, patentemente, como resto una relación entre la percepción y el percepto (como igualmente entre el agrado y lo grato), ... ${ }^{12}$.

Del mismo modo, cuando Husserl quiere poner el énfasis en que el noema no resulta alterado por su puesta entre paréntesis, incluye entre las cosas que se ponen entre paréntesis no sólo a la cosa individual, aislada, que percibimos, sino al mundo entero:

El pleno noema... no resulta afectado por la exclusión de la realidad del árbol ni del mundo entero. ${ }^{13}$

Más adelante, parece mantener que el noema abarca en su totalidad al horizonte. Por ejemplo, escribe en un manuscrito:

La estructura horizonte con sus niveles y penetraciones es el cañamazo noemático del sentido y validez, a través de la cual la objetividad se mienta en cada caso para nosotros... ${ }^{14}$

Aunque Husserl no es explfcito respecto a la cuestión terminológica -cuántas de nuestras anticipaciones comprehende el noema, todas o solo algunas-, se expresa, sin embargo, con claridad respecto al punto que realmente nos interesa. En todos sus escritos desde Ideas, 
Husserl dice claramente que cuando apuntamos hacia un objeto apuntamos al mismo tiempo hacia el mundo entero al que el objeto pertenece.

\section{EL "ICEBERG"}

El horizonte es de crucial importancia para el concepto husserliano de justificación, que discutiremos luego. Lo que es de singular relevancia es la naturaleza oculta del horizonte. Como apuntamos anteriormente, el horizonte es aquello a lo que no atendemos. Normalmente, como en el caso del suelo de la habitación del ejemplo anterior, ni tan siquiera habiamos reflexionado sobre él. Incluso, no podemos recordar cuando adquirimos por primera vez la "creencia" o "anticipación" correspondiente. De acuerdo con Husserl, no puede haber ninguna ocasión en la que juzgemos realmente que hay un suelo en una habitación particular. Sin embargo, nosotros hemos tenido que "anticipar" un suelo; no en el sentido de esperar uno conscientemente, sino en el de quedar estupefactos si al entrar en una habitación no hay ninguno. En este ejemplo, serfamos capaces de decir fácilmente lo que faltaba, en otros casos nuestras "anticipaciones" son tan imperceptibles que sólo podríamos sentir que algo ha fallado sin que fueramos capaces de decir el qué.

Palabras como "creencia" y "anticipación" no son con mucho las más apropiadas puesto que sugieren algo consciente y tematizado. Tanto el inglés como el alemán carecen de palabras para nuestros presentes objetivos: Husserl usa las palabras "antizipieren", "hinausmeinen" y tambiên "vorzeichnen". 
La problemática del mundo de la vida está intimamente ligada a la distinción entre la actitud natural y la transcendental, o fenomenológica, que Husserl introdujo en 1906/07. La primera mención de la problemática para la que más tarde introdujo el término 'Lebenswelt' tuvo lugar poco después en sus lecciones "Grundprobleme der Phänomenologie" (1910/11), es decir, con anterioridad a Ideas. Husserl comienza estas lecciones con una amplia discusion sobre "Die natürliche Einstellung und der 'natürliche Weltbegriff' ". Husserl nos dice aqui:

Podría mostrarse también que los intereses filosoficos de la más alta dignidad requieren una descripción completa y comprensiva del así llamado "concepto natural de mundo", el de la actitud natural; por otro lado, también, que una descripción adecuada y profunda de este tipo no se consigue fácilmente sino que, por el contrario, requeriría reflexiones de enorme dificultad. ${ }^{15}$

Husserl, según su propio testimonio, toma prestada aquí la expresión "naturlicher Weltbegriff" de Avenarius, sobre quien discutirá más adelante en la lección ${ }^{16}$. Husserl describe este mundo siguiendo a Avenarius en un manuscrito de 1915 del siguiente modo:

Todas las opiniones, justificadas e injustificadas, popularmente, supersticiosamente, científicamente, todo lo que mantiene una relación con el mundo ya pre-dado...toda teoría se relaciona con este dado inmediato y puede tener un sentido legítimo sólo cuando forma pensamientos que no colisionan con el sentido general de lo inmediatamente dado. No teorizar puede colisionar con este sentido. ${ }^{17}$

En años sucesivos, Husserl vuelve repetidas veces sobre el mismo tema y sobre otros relacionados con este, haciendo uso de 
diferentes denominaciones que, algunas veces, aluden a otros filosofos, entre ellos Nietzsche, que propusieron ideas similares. Husserl usa bastante a menudo la expresión de Avenarius "naturliche Welt". En un manuscrito de 1917, que según parece es el primer texto donde aparece la palabra 'Lebenswelt', introduce esta nueva palabra como equivalente a la primera expresión:

El mundo de la vida es el mundo natural- en la actitud de persecución natural de la vida, estamos viviendo sujetos funcionantes involucrados en el círculo de otros sujetos funcionantes. ${ }^{18}$

Gradualmente, durante los años 20 y especialmente en los años 30 , el mundo de la vida se convierte en el tema central de los escritos husserlianos hasta su elaboración definitiva en La Crisis (1936). Uno de los objetivos fundamentales de esta última obra de Husserl fue el de ofrecer a través de la noción de mundo de la vida un nueo y mejor acceso a la fenomenología. El mundo de la vida es para Husserl nuestro mundo natural, el mundo en el que vivimos y en el que estamos imbuidos en nuestras actividades cotidianas. Una de las principales metas de la fenomenologia es la de hacernos reflexionar sobre este mundo, hacernos ver que nosotros lo constituimos. Mediante un peculiar modo de reflexión, la "reducción fenomenológica", la fenomenología nos transportará desde nuestra actitud natural, donde somos devorados por el mundo circundante, a la actitud fenomenológica, transcendental, donde nos concretamos en los noémata de nuestros actos, en nuestro estructurar la realidad. Es decir, somos nosotros quienes configuramos la "puesta entre paréntesis" que Husserl describiera en aquel primer pasaje de Ideas que cité en la sección dedicada al noema, el horizonte y el mundo. 


\section{PREDATITUD E INTERSUBJETIVIDAD}

En el pasaje del manuscrito de 1915 que cité anteriormente, Husserl nos dice que el mundo está pre-dado [vorgegeben]. Este punto se discute también en Ideas, donde Husserl apunta que:

De este modo me encuentro en todo momento de la vigilia, y sin poder evitarlo, en relación consciente al uno y al mismo mundo, bien que cambiante de contenido. Este mundo está persistentemente para mí "ahf delante", yo mismo soy miembro de él, pero no está ahr como un mero mundo de cosas, sino, en la misma forma inmediata, como un mundo de valores y de bienes, un mundo práctico. ${ }^{19}$

Y algunas páginas más adelante:

Yo encuentro constantemente ahí delante, como algo que me hace frente, la realidad [Wirklichkeit] espacial y temporal una, a que pertenezco yo mismo, como todos los demás hombres con que cabe encontrarse en ella y a ella están referidos de igual modo ${ }^{20}$

En las mismas secciones de Ideas, Husserl recalca la naturaleza participativa, intersubjetiva de este mundo. Lo hace muy especialmente en el $\S 29$, que titula "Los 'otros' sujetos-yos y el mundo circundante natural e intersubjetivo". Nos dice aqui:

...concibo su mundo circundante y el mío como siendo objetivamente un mismo mundo, del que todos nos limitamos a tener conciencia de diverso modo... Acerca de todo esto nos entendemos con los prójimos, poniendo en común una realidad espacial y temporal objetiva.. ${ }^{21}$

Las mismas ideas de predatitud e intersubjetividad se repiten casi con las mismas palabras cuando Husserl presenta el mundo de la vida en La Crisis; por ejemplo, en el $\S 37$ donde dice: 
El mundo de la vida es -...- para nosotros lo que en la vigilia siempre está ahí, lo que existe con anterioridad para nosotros, el "suelo" de toda praxis ya sea teórica o extrateórica. El mundo está pre-dado para nosotros, en la vigilia, y siempre para todo sujeto interesado prácticamente, no accidentalmente sino siempre necesariamente, como campo universal de toda praxis real y posible, como horizonte ${ }^{22}$

Tanto en La Crisis como en Ideas, Husserl pone el énfasis en la intersubjetividad. Así, por ejemplo, en el $\S 47$ de La Crisis:

Por esto, en general, el mundo existe no sólo para un hombre aislado sino para la comunidad de los hombres, y esto se debe al hecho de que incluso lo que es estrictamente perceptual es comunitario. ${ }^{23}$

\section{¿CONSTITUYE EL MUNDO DE LA VIDA UN DESARROLLO TARDIO DEL PENSAMIENTO HUSSERLIANO?}

Como he mencionado en la introducción, se piensa a menudo que el mundo de la vida supone la mayor interrupción en el desarrollo del pensamiento husserliano desde el primer Husserl de ideas hasta el último de La Crisis. Uno de los máximos exponentes de dicha opinión es David Carr, quien escribe en su Interpreting Husserl: ${ }^{24}$

Es posible pensar que el concepto de mundo de la vida no representa en realidad nada nuevo en el pensamiento de Husserl, ... Pero, como revela la exposición de Husserl, este concepto pone en juego características que lo hacen distinto, en más de un aspecto, de lo que había hecho antes.

Una de estas características es la prominencia de la noción de Vorgegebenheit o predatitud...

Una segunda característica, que distingue esta concepción del mundo percibido de La Crisis de anteriores concepciones, es que el mundo es insistentemente descrito como público o intersubjetivo: está 'pre-dado 
como existiendo para todos en común', como el "mundo común a todos nosotros".

Los pasajes de las primera obras y manuscritos husserlianos que he citado, ponen de manifiesto que hay una considerable continuidad entre el primer y segundo Husserl respecto al mundo de la vida y a las dos características de éste mencionadas por Carr. Lejos de poner el énfasis en estas diferencias, yo quisiera acentuar la continuidad en el dasarrollo de Husserl. Cuando tratamos de entender a Husserl encuentro especialmente relevante apuntar la intima conexión entre sus nociones de noema, horizonte y mundo de la vida.

\section{¿UNO O MUCHOS MUNDOS DE LA VIDA?}

Sin embargo, nos encontramos ahora con un problema. Husserl parece contradecirse a sí mismo en relación al número de mundos de la vida. Por lo que hasta ahora se ha dicho, esperariamos que todos nosotros tuviéramos diferentes mundos de la vida dependiendo de nuestro nivel cultural, nuestras experiencias pasadas, y así sucesivamente. Husserl también se expresa en estos términos, por ejemplo, en sus lecciones sobre Phanomenologische Psychologie de 1925:

No compartimos el mismo mundo de la vida con el resto de la gente, no toda la gente "en el mundo" tiene en común con nosotros todos los objetos que constituyen nuestro mundo de la vida y que determinan nuestra actividad personal y nuestras aspiraciones incluso cuando ellos se avienen en una eventual asociación con nosotros, como pueden hacer siempre ${ }^{2 s}$

Esto encaja perfectamente con la afirmación de Husserl de que nuestro mundo de la vida cambia del mismo modo que nuestras opiniones. Por lo que, como veremos después, el desarrollo de la 
ciencia cambia nuestro mundo de la vida. Por otro lado, sin embargo, Husserl insiste también en que sólo hay un mundo de la vida:

Por otro lado, el mundo no existe como una entidad, como un objeto, sino que existe con una unidad tal que el plural no tiene sentido cuando lo aplicamos a él. Cada plural y cada singular desgajado de él presupone ya al mundo como horizonte. ${ }^{26}$

Por supuesto podemos concluir que Husserl se contradice en este punto y que podemos optar indiferentemente por una de las dos alternativas: o bien una pluralidad de mundos de la vida o sólo uno. Sin embargo, cuando interpretamos benévolamente a Husserl no existe tal contradicción y podemos aceptar ambos puntos de vista como expresando importantes características del mundo de la vida. Para leer a Husserl de este modo tenemos sólo que retener en la mente el tema general y recurrente de toda la fenomenología, a saber; que un único y mismo objeto puede aparecer de muy diversas maneras. Veamos, pues, en qué sentido cabe hablar de un único mundo de la vida.

Hay sólo un mundo de la vida en el siguiente sentido: el mundo que se le aparece a una persona en sus múltiples experiencias es siempre el mismo, y éste es también el mundo que se le aparece a los demás sin que importe cuándo y dónde vivan. Nuestras concepciones de este mundo pueden diferir $y$, en este sentido, todos vivimos en diferentes mundos. Pero diferente no es lo mismo que distinto. El mundo en el que vive cada uno de nosotros es único, pero se manifiesta a cada uno de nosotros de modos diversos.

Que el mundo se nos presente de modo diferente a cada uno de nosotros, es algo que no merece mayor atención. Pero la unicidad de este mundo es un aspecto muy importante en la filosofía husserliana. La idea de que sólo hay un mundo en el que todos vivimos supone a su vez las de intersubjetividad y comunicación. Ambos, intersubjetividad y comunicación, tienen por base la idea de que sólo hay un 
mundo del cual tenemos experiencia desde nuestras diversas perspectivas. Otra importantísima característica de un único mundo de la vida fue apuntada en la última frase del texto de Husserl que he citado más arriba: "Cada plural y cada singular presupone ya al mundo como horizonte". Este tema es fundamental en la discusión husserliana acerca del mundo de la vida y aclarada en posteriores fragmentos de sus obras. El más claro de ellos es el $\S 37$ de La Crisis, que cité antes en conexión con la predatitud del mundo de la vida.

Según Husserl, cuando nos posicionamos con respecto a la existencia de un objeto, a la verdad o falsedad de un enunciado; cuando valoramos algo positiva o negativamente y proponemos metas y propósitos para nuestras vidas; todo esto ocurre dentro de un mundo de la vida y, lo que es más importante, sólo en virtud del mundo de la vida. El mundo de la vida es, en expresión husserliana, el fundamento de la validez [Geltungsfundierung] o el suelo [Boden] para este posicionarse $e^{27}$. Esta está "ya ahl" sirviendo de "unhintergehbar" repertorio para todos nuestros posicionamientos. Por ejemplo, podemos siempre afirmar o negar la existencia de un objeto, pero no podemos negar la existencia del mundo. Podemos tratar de hacerlo, pero en este caso nuestras palabras carecerfan de "behauptende Kraft" por usar la expresión fregeana. Volveremos este punto en la sección final de este artículo donde se discutirá el papel del mundo de la vida en la justificación.

\section{CIENCIA Y MUNDO DE LA VIDA}

Existe controversia en los estudios husserlianos sobre la relación entre el mundo de la vida y las ciencias. Muchos intérpretes de Husserl pretenden encontrar una oposición entre el mundo de la vida 
y las ciencias. Sin embargo, un desdén tal hacia las ciencias no encaja con la formación académica de Husserl y su continuo interés por las matemáticas y la ciencia. Tampoco concuerda fácilmente con los textos, que nos ofrecen una imagen diferente y más intrigante. De acuerdo con Husserl, el mundo de la vida y las ciencias están intimamente ligados de tres formas diferentes:

1. El mundo de la ciencia forma parte del mundo de la vida. Esto se ve más clara y explícitamente en Experiencia y Juicio, donde Husserl afirma:

...también nos pertenece, al mundo tal como nos ha sido pre-dado a los adultos de nuestro tiempo, cada cosa que la ciencia natural contemporánea ha añadido como determinaciones de lo que existe. $E$ incluso si no estamos interesados personalmente en la ciencia natural e incluso si no sabemos nada de sus resultados, todavía, lo que existe nos está pre-dado con anterioridad como determinado de tal modo que como mínimo lo concebimos como siendo, en principio, científicamente determinable. $^{28}$

Afirmaciones similares se encuentran también en otros escritos de Husserl, por ejemplo, en La Crisis:

El mundo científico -la teoría sistemática-... como todo mundo de fines "pertenece" al mundo de la vida ${ }^{29}$

2. Las proposiciones cientificas adquieren sus significados en el seno del mundo de la vida. Husserl lo puso ya de manifiesto en el

manuscrito de 1915 que cité más arriba en la sección dedicada a el mundo de la vida y el mundo natural: 
Todas las opiniones, justificadas e injustificadas. popular, supersticiosa o científicamente, todo lo relacionado con el mundo ya pre-dado... toda teorfa se relaciona con este dado inmediato y puede tener un sentido legítimo sólo cuando forma pensamientos que no colisionan con el sentido general de lo inmediatamente dado. No teorizar puede colisionar con este sentido..$^{30}$

3. Las ciencias encuentran su justificación através del mundo de la vida. Existe cierta relación entre este punto y el punto 1 anterior; las ciencias encuentran su justificación por el hecho de pertenecer al mundo, y al mismo tiempo, pertenecen al mundo de la vida porque están concebidas como descripción del mundo, como pretendiendo ser verdaderas:

Ante la posibilidad de que el resultado peculiar de nuestra ciencia objetiva moderna no pueda todavía ser comprendido, nada cambia el hecho de que el mundo de la vida posee una validez, emergiendo de las actividades particulares, y que pertenece él mismo a la concreción del mundo de la vida ${ }^{31}$

Y de modo análogo:

Todos estos resultados teóricos tienen el carácter de validez para el mundo de la vida, añadiendo ya ellos mismos como tales para su propia composición y perteneciendo a él de antemano como horizonte de posibles efectuaciones para el desarrollo de la ciencia. El mundo de la vida concreto, entonces, es el suelo fundante [der grindende Boden] del mundo "científicamente verdadero" y al mismo tiempo abarcan a este en su propia concreción universal. ${ }^{32}$

\section{JUSTIFICACION ULTIMA}

Esto nos lleva al tema final de este artículo: el papel del mundo de la vida en la justificación. En un articulo anterior he argüido 
-contra la interpretación tradicional de Husserl como fundamentalistaque Husserl sostiene un punto de vista, tanto en ética como en ciencias, similar al de Goodman y Rawls ${ }^{33}$. Una opinión está justificada cuando mantiene un "equilibrio reflexivo" con la doxa de nuestro mundo de la vida. Esto es válido también para las matemáticas:

...la evidencia matemática tiene su fuente de significado y legitimidad en la evidencia del mundo de la vida. ${ }^{34}$

El mayor problema al que se enfrenta para muchos esta idea de justificación es "¿Cómo podemos apelar a la subjetiva y relativa doxa para que nos provea de una justificación para algo? Podría servir como coadyubante para reconciliar desacuerdos, pero, ¿cómo puede servir de justificación? La respuesta de Husserl pasa por poner de manifiesto la carencia de otra forma de justificación asf como lo satisfactorio de esta primera:

Lo realmente primero es la intuición "meramente subjetiva relativa" de la vida precientífica mundana. Para nosotros, sin duda, este "meramente" tiene, como si de una vieja herencia se tratase, la coloración desautentificadora de la doxa. En la vida precientífica misma no hay nada de esto; allí es un campo de buena verificación y, sobre esta base, de conocimientos predicativos plenamente verificados y de verdades que son exactamente tan seguras como necesaria es para los proyectos prácticos de la vida a los que determina su sentido. Esta desautentificación, que aplican a cada cosa "meramente subjetivo-relativa" aquellos científicos que persiguen el ideal moderno de objetividad, no cambia nada de su propia manera de ser como tampoco cambia el hecho de que el científico mismo tiene que estar satisfecho con este campo al que siempre ha recurrido y al que inevitablemente tiene que recurrir ${ }^{35}$ 
Hasta el momento, todo esto es una suposición. Sin embargo, Husserl elabora sus puntos de vista en otras partes de su obra. Su observación clave, que considero una contribución intrigante a nuestra discusión contemporánea sobre la justificación última, es que las "creencias", "expectaciones" o "aceptaciones" a las que recurrimos en último término no están tematizas y, en la mayoría de los casos, no lo han estado nunca. Cada pretensión de validez y de verdad descansa sobre este "iceberg" de aceptaciones no tematizadas y prejuiciosas de las que hablamos anteriormente. Se podría pensar que esto empeora todavia más las cosas. No sólo recurrimos a algo que es inseguro sino a algo sobre lo que todavia no hemos reflexionado $y$, por tanto, nunca puesto a prueba concienzudamente. Husserl argumenta, sin embargo, que es la naturaleza no tematizada del mundo de la vida lo que lo convierte en fundamento para la justificación. "Aceptación" y "creencia" no son aptitudes que decidamos tener mediante un acto de decisión judicativa. Aquello que aceptamos, y el fenómeno mismo de la aceptación, son ingredientes de nuestro mundo de la vida, y no hay forma de partir de cero, o "evadirse mediante la preocupación por la aporía y la argumentación que fomentara Kant o Hegel, Aristóteles o Santo Tomás" ${ }^{136}$. Sólo el mundo de la vida puede ser último tribunal de apelación:

Sólo por ello puede alcanzarse esa comprensión última del mundo detrás de la cual, puesto que es última, no hay nada más que pueda ser significativamente investigado, nada más que comprender. ${ }^{37}$

Universidad de Oslo y Stanford University 


\section{NOTAS}

1 El origen de este artículo es un proyecto de investigación sobre el que estuve trabajando en calidad de miembro del Wissenschafiskolleg (Berlín) durante 1989-90. Reconozco agradecido la ayuda prestada.

2 Georg Simmel, Die Religion (Die Gesellschaft, Sammlung sozialpsychologischer Monografien, ed. Martin Buber), Rütten \& Loening, Frankfurt a. M., 2. ed. 1912, pág.13. Sirnmel concibe el mundo de la vida como "aufgebaut", y también escribe que "das religiöse Lebenschafft die Welt" (pág. 12, el subrayado es mio). Hugo von Hofmannsthal usó el ténnino incluso antes es su introducción de la edición de Insel de Las Mil y Una Noches (1908), donde escribe: "...was wären diese Gedichte, was wären sie uns, wenn sie nicht aus einer Lebenswelt hervorstiegen. Unvergleichlich ist diese Lebenswelt, und durchsetzt von einer unendlichen Heiterkeit, ... die alles durcheinanderschlingt, alles zueinanderbringt..." (Prosa II, Frankfurt a.M., 1951, 319).

3 Maurice Merleau-Ponty, Phénoménologie de la perception, Gallimard, París, 1945, pág 108, n., donde Merleau-Ponty hace referencia al segundo volumen de Ideas. En el Prefacio a la Phénoménologie de la perception (p. VII) Merleau-Ponty menciona que también había estudiado el manuscrito husserliano de La Crisis. Vid. H.L. van Breda, "Maurice Merleau-Ponty et les Archives-Husserl à Louvain", Revue de métaphysique et de morale, LXVII (1962), 410-430. Vid. tambiến la traducción inglesa de David Carr de La Crisis, p xxx, notas 20 y 21, y Mafred Sommer, Lebenswelt und Zeitbewusstsein, Suhrkamp, Frankfurt, 1990, p.84, n.70.

4 Vid. mi "Intentionality and behaviorism". En L.J. Cohen, J. Los, H. Pfeiffer y Klaus-Peter Podewski, Eds., Proceedings of the 6th Intemational Congress of Methodology and Philosophy of Science, Hannover, August 22-29, 1979, Amsterdam: North-Holland, 1982, pp. 553-569.

5 Ideas, \$135, edición original, p. 281, Husserliana III, 1, 313. 16-20. Traducción de Gaos, pág. 324.

6 Carta citada por Iso Kem en su Husserl und Kant. Eine Untersuchung aber Husserls Verhaltnis zu Kant und zum Neukantianismus (Phenomenologica 16). Martinus Nijhoff, The Hague, 1964, p. 276 nota.

7 Husserl, Prefacio a la traducción inglesa de Ideas, Allen \& Unwin, London, 1931. Versión alemana en Husserliana $\mathrm{V}, 152,32-153.5$.

8 Erfahrung und Urteil, 21 , pag. 96.

9 Erfahrung und Urteil, \& 8, pp. 28-29.

10 Este ejemplo surgió en unas converaaciones que mantuve con David Wellbery.

11 "Husserl and Heidegger on the role of actions in the constitution of the world". En E. Saarinen, R. Hilpinen, I. Niiniluoto y M. Provence Hintikka, Eds, Essays in Honour of Jaakko Hintikka, Dondrecht, Holland: Reidel, 1979, 365-378.

12 Ideas, $\$ 88$, pp. 182-183 de ln edición general, Husserliana III, 1, 226.2-4. Gaos, pág. 214. 

236.

Ideas, 897 , p. 202 de la edición original, Husserliana III, 1, 226.2-4. Gaos, pág.

14 Manuseript B II 9, p.146: "Die Horizonstruktur in ihren Stufen und Durchdringungen ist das noematische Sinn- und Geltungsgerüst, in dem Objektivität für uns jeweils vermeinte ist...". Citado por Alwin Diemer en Edmund Husserl. Versuch einer systematischen Darstellung seiner Phänomenologie (Monogrophien zur philosophiachen Forschung, Band XV), Anton Hain, Meisenheim am Glan, 2. ed., 1965, p. 68, n. 29.

15 Husserliana XIII, 124.34-125.7.

16 Avenarius, Der menschliche Weltbegriff, Leipzig, 1891.

17 Husserliana XIII, 196.22-34.

18 Husserliana IV, 375.31-33, el manuserito data de 1917 pero fue copiado durante la primera mitad de los años veinte y es posible que la palabra "Lebenswelt" se introdujera entonces.

19 Ideas, § 27, Husserliana III, 1, 58.13-19. Trad. de Gaos, pág. 66.

20 Ideas, \$ 30, Husserliana III, 1, 61.15-18. Trad.de Gaos, pág. 69.

21 Ideas, \$29, Husserliana III, 1, 60.16.26. Gaos, pág. 68.

22 Krisis, \$ 37, Husserliana VI, 145.24-32.

23 Krisis, \& 47, Husserliana VI, 166, 19-22.

24 David Carr, Interpreting Husserl (Phaenomenologica 106), Nijhoff, Dondrecht, 1987, pp. 232-34.

25 Husserliana, IX, 496.40-45.

26134,143 y 462 .

27 Krisis, Husserliana VI, 146.14-17.

28 Erfahrung und Urteil, \$ 10, p.39.

29 Krisis, Beilage XVII, Husserliana VI, 460.23-27.

30 Husserliana XIII, 196.22-34.

31 Krisis, \$ 34f, Husserliana VI, 136.18-22.

32 Krisis, 34e, Husserliana VI, 134.13-19.

33 "Husserl on evidence and justification". En Robert Sokolowski (Ed.), Edmund Husserl and the Phenomenological Tradition: Essays in Phenomenology, The Catholic University of America Press, Washington, 1988, pp. 107-129.

34 Krisis, \& 36, Husserliana VI, 143.29-30.

35 Krisis, \& 34a, Husserliana VI, 127.31-128.10.

36 Krisis, \$ 34e, Husserliana VI, 134.35-37.

37 Formale und Transzendentale Logik, $\$ 96 \mathrm{~b}$, Husserliana XVII, 249.18-20. 